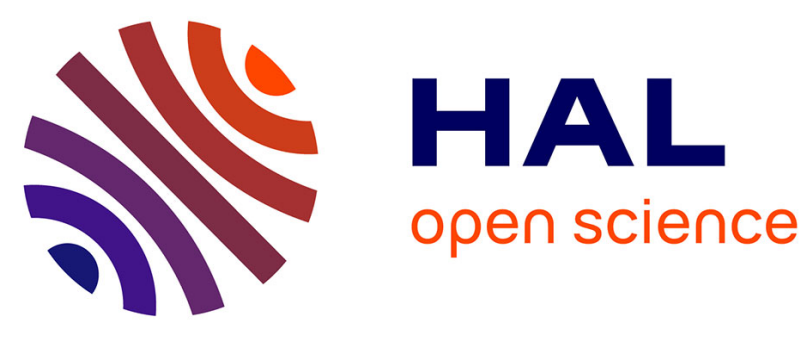

\title{
Leukoencephalopathy and conduction blocks in PLEKHG5-associated intermediate CMT disease
}

Nguyen Thi Huong, Rocio-Nur Villar-Quiles, Van Thuy Le, Sarah

Leonard-Louis, Lilia Laddada, Bruno Francou, Thierry Maisonobe, Hamid

Azzedine, Tanya Stojkovic, Thi Trang Nguyen

\section{To cite this version:}

Nguyen Thi Huong, Rocio-Nur Villar-Quiles, Van Thuy Le, Sarah Leonard-Louis, Lilia Laddada, et al.. Leukoencephalopathy and conduction blocks in PLEKHG5-associated intermediate CMT disease. Neuromuscular Disorders, 2021, 10.1016/j.nmd.2021.06.004 . hal-03285057

\section{HAL Id: hal-03285057 \\ https: / hal.sorbonne-universite.fr/hal-03285057}

Submitted on 13 Jul 2021

HAL is a multi-disciplinary open access archive for the deposit and dissemination of scientific research documents, whether they are published or not. The documents may come from teaching and research institutions in France or abroad, or from public or private research centers.
L'archive ouverte pluridisciplinaire HAL, est destinée au dépôt et à la diffusion de documents scientifiques de niveau recherche, publiés ou non, émanant des établissements d'enseignement et de recherche français ou étrangers, des laboratoires publics ou privés. 
1 TITLE: Leukoencephalopathy and conduction blocks in PLEKHG5-associated

2 intermediate CMT disease.

3 AUTHOR NAMES AND AFFILIATIONS

4 Rocio-Nur Villar-Quiles ${ }^{a, b}$, Van Thuy Le ${ }^{c}$, Sarah Leonard-Louis ${ }^{a}$, Nguyen Thi

5 Trang $^{d}$, Nguyen Thi Huong ${ }^{c, e}$, Lilia Laddada ${ }^{f, g}$, Bruno Francou ${ }^{f}$, Thierry

6 Maisonobe $^{h}$, Hamid Azzedine ${ }^{i}$, Tanya Stojkovic ${ }^{a, b}$

7 aReference Center for Neuromuscular Disorders, APHP, Pitié-Salpêtrière 8 Hospital, Paris, France

bSorbonne Université - Inserm UMRS974, Centre de Recherche en Myologie, GH Pitié-Salpêtrière, Paris, France

'Neurology department, Hanoi Medical University Hospital, Hanoi, Viet Nam

${ }^{d}$ Genetics department, Hanoi Medical University Hospital, Hanoi Medical University Hanoi, Viet Nam

e Vinmec International Hospital, Hanoi, Vietnam

fDepartment of Molecular Genetics Pharmacogenomics and Hormonology, APHP, Bicêtre Hospital, Paris, France,

gPlateforme d'expertise maladies rares AP-HP. Université Paris-Saclay, Le Kremlin Bicêtre, France

hDepartment of Neurophysiology, APHP, Hôpital Pitié Salpêtrière, Paris, France iDepartment of Pathology and Neuropathology, AMC, Amsterdam, Netherlands

\section{CORRESPONDING AUTHOR}
Dr Tanya Stojkovic

Centre de Référence des maladies neuromusculaires Nord / Est / lle-de-France

Groupe Hospitalier Pitié-Salpêtrière

47-83 boulevard de l'Hôpital

75651 Paris Cedex 13, France

e-mail address: tanya.stojkovic@aphp.fr

DECLARATIONS OF INTEREST: none 
Biallelic variants in PLEKHG5 have been reported so far associated with different clinical phenotypes including Lower motor neuron disease (LMND) [also known as distal hereditary motor neuropathies (dHMN or HMN) or distal spinal muscular atrophy (DSMA4)] and intermediate Charcot-Marie-Tooth disease (CMT). We report four patients from two families presenting with intermediate CMT and atypical clinical and para-clinical findings. Patients presented with predominant distal weakness with none or mild sensory involvement and remain ambulant at last examination (22-36 years). Nerve conduction studies revealed, in all patients, intermediate motor nerve conduction velocities, reduced sensory amplitudes and multiple conduction blocks in upper limbs, outside of typical nerve compression sites. CK levels were strikingly elevated (1611-3867 U/L). CSF protein content was mildly elevated in two patients. Diffuse bilateral white matter lesions were detected in one patient. Genetic analysis revealed three novel frameshift variants c.1835_1860del and c.2308del (family 1) and c.104del (family 2). PLEKHG5-associated disease ranges from pure motor phenotypes with predominantly proximal involvement to intermediate CMT with predominant distal motor involvement and mild sensory symptoms. Leukoencephalopathy, elevated $\mathrm{CK}$ levels and the presence of conduction blocks associated with intermediate velocities in NCS are part of the phenotype and may arise suspicion of the disease, thus avoiding misdiagnosis and unnecessary therapeutics in these patients.

KEY WORDS: intermediate CMT; PLEKHG5; conduction block; leukoencephalopathy; CSF protein; elevated CK 
55 FUNDING: This research did not receive any specific grant from funding agencies 56 in the public, commercial, or not-for-profit sectors.

\section{1. INTRODUCTION}

58

Hereditary motor and sensory neuropathies, classically known as Charcot-MarieTooth disease (CMT), represent the commonest group of inherited neuromuscular diseases [1]. These clinically and genetically heterogeneous disorders are usually characterized by progressive distal muscle atrophy and weakness, foot deformities and sensory loss.

Historically, based on electrophysiological findings, CMT were classified into demyelinating forms (CMT1) when upper limb motor nerve conduction velocity (MNCV) is significantly decreased $(<38 \mathrm{~m} / \mathrm{s}$ ) and axonal forms (CMT2) with normal or slightly reduced MNCV and significantly reduced amplitudes of motor and sensory nerve action potentials. Additionally, intermediate CMT includes forms with MNCV between those of typical CMT1 and CMT2 (usually between 35 and $45 \mathrm{~m} / \mathrm{s}$ )[2]. Nerve biopsies in these intermediate forms show the coexistence of axonal (degeneration of large axons and regenerative sprouting) and demyelinating (thin or absent myelin sheaths, onion bulb formation) abnormalities.

Recessive mutations in Pleckstrin Homology And RhoGEF-domain-containing G5 gene (PLEKHG5), initially reported in one consanguineous family with earlyonset severe lower motor neuron disease (or Distal Spinal Muscular Atrophy [DSMA4]) [3], have been also formally associated with intermediate CMT [4,5]. More recently, cases with either pure lower motor neuron disease (LMND), distal and proximal neuropathy with mild sensory involvement or intermediate-CM have been documented [6-8]. Here, we report two additional families carrying novel 
PLEKHG5 mutations presenting with an intermediate CMT phenotype associated with atypical findings, including leukoencephalopathy, conduction blocks on electrophysiological studies and highly elevated creatine kinase (CK), thus expanding the phenotypical spectrum of the disease.

84

\section{PATIENTS AND METHODS}

86 Four patients ( 3 women and 1 man) from two unrelated families were examined

87 by one of the authors. Nerve conduction studies and electromyography were performed using standard techniques [9]. Definite conduction blocks were defined by a drop in more than $50 \%$ in distal compound muscle action potential (CMAP) amplitude, while probable conduction blocks were defined by a drop of $30-50 \%$ distal CMAP drop [10]. Brain MRI was performed in two patients while muscle biopsy was conducted in one and processed for standard histological and immunochemical studies [11].

DNA samples were extracted from peripheral blood samples and whole exome sequencing (family

1) or next generation sequencing-based neuropathy multigene panel (family 2) were performed. Sanger sequencing confirmed the selected variants we identified. They were reported according to Human Genome Variation Society recommendations (http://varnomen.hgvs.org).

GnomAD database (http://gnomad.broadinstitute.org) was used to search for the variants' allele frequencies.

101 Informed consent was obtained from all patients, in agreement with local ethic committees and with the 1964 Helsinki declaration and its later amendments

\section{RESULTS}




\subsection{Clinical findings}

106 Family 1

107 The two siblings from a non-consanguineous Vietnamese family (Figure 1A).

108 They have normal neuropsychological development. Patient III.5 presented with

109 distal lower limbs weakness and numbness first noted in adolescence with a

110 slowly progressive course. Examination revealed a steppage gait, mild distal

111 muscle atrophy, distal weakness (foot dorsiflexion 3/5), distal hypoesthesia in a

112 stocking-and-glove distribution and abolished deep tendon reflexes (DTRs). The

113 patient was able to walk without aid at last examination (Table 1). Patient III.4

114 had a more severe form with severe distal and proximal involvement of four limbs

115 (3/5 at distal and 4/5 at proximal UL, $2 / 5$ distal $3 / 5$ at proximal LL) but independent

116 ambulation was preserved. She had pes cavus and Achilles contractures.

117 Nerve conduction studies (NCS) disclosed reduced MNCVs in four limbs,

118 conduction blocks ( $>50 \%$ ) in upper limbs (Figure $2 \mathrm{~A}$ ) affecting median and ulnar

119 nerves in the forearm out of typical nerve compression sites for patient III.4 while

120 for patient III.5 there was a definite conduction block affecting the median nerve

121 and a probable conduction block (-34.6\%) affecting the ulnar. Most sensory nerve

122 action potentials (SNAPs) were abolished. Needle EMG of gastrocnemius muscle

123 from patient III.4 revealed giant MUAPs and poor recruitment indicating a chronic

124 neurogenic pattern but also the presence of complex repetitive discharges

125 (CRDs) (Figure 2C-D).

126 Interestingly, cerebrospinal fluid (CSF) analysis performed during the initial

127 diagnostic workup in patient III.5 revealed raised CSF protein level up to $0.70 \mathrm{~g} / \mathrm{L}$

128 (normal $0.15-0.45 \mathrm{~g} / \mathrm{L}$ ). Patient III.5 had also significantly raised serum creatine 
129 kinase $(\mathrm{CK})$ levels (1611 U/L). Cerebral MRI from the same patient was normal

130 aside from one millimetric white matter hyperintensity on T2-weighted images.

132 Family 2

133 The two siblings from family 2 were born to consanguineous marriage of parents

134 from Ivory Coast. Patient II.4 presented since early childhood with difficulty

135 running, poor sports performance and frequent falls. Subsequent progressive 136 distal upper and lower limb weakness was noted. Examination revealed 137 abolished DTRs in LL, predominantly distal weakness (UL 3/5, LL 4/5) but also 138 proximal UL (3/5) weakness. Distal reduced vibration sensation was noted with 139 no abnormalities on pinprick test. Her sister presented at age 20 with subacute 140 onset LL weakness. Clinical examination revealed cavovarus feet, hammer toes, 141 distal LL weakness (4/5) and mild proximal weakness (mainly hip extension 142 weakness), abolished DTRs and normal sensory examination. Both patients were 143 able to walk independently at last examination, but gait perimeter was limited for 144 patient II.4.

145 NCS from both siblings disclosed reduced MNCVs in four limbs as well as 146 moderately reduced SNAPs with intermediate sensory NCVs, especially for

147 patient II.4. Interestingly, probable conduction blocks in UL out of sites of potential 148 nerve compression (i.e. median nerve, Figure2B) were found for patient II.4 and 149 definite conduction blocks in the fibular nerve were noted in both cases. For 150 patient II.6, 20 and $24 \%$ drop in distal CMAP amplitude were detected in median and ulnar nerves respectively, out of sites of potential nerve compression. Needle

152 EMG disclosed high-amplitude motor unit potentials ( $\geq 5 \mathrm{mV}$ ) with reduced 153 recruitment without spontaneous activity, suggesting a chronic neurogenic 
154 process. Interestingly, CSF analysis of both patients revealed a slightly raised

155 protein content $(0.68 \mathrm{~g} / \mathrm{L}$ in patient 11.5 and $0.53 \mathrm{~g} / \mathrm{L}$ in II.6).

156 Similarly to patient III.5, patient II.4 had very raised CK levels (3867 U/L) (Table

157 1). Moreover, cerebral MRI disclosed posterior bilateral white matter lesions

158 (Figure 2E). Given the highly raised CK levels, a deltoid muscle biopsy was

159 performed, revealing fiber size variability, sparse necrotic fibers and mild fibrosis.

160 A genetic analysis of limb-girdle muscle dystrophies (LGMD)-causing genes by 161 next-generation sequencing (NGS), including LAMA2, was negative. A metabolic 162 workup (thyroid hormones, transferrin electrophoresis, phytanic acid, 163 arylsulfatase, galactocerebrosidase, cholestenol and long chain fatty acids 164 dosages and apolipoprotein profiles) conducted for patient II.4 was normal.

\section{$165 \quad 3.2$ Genetic findings}

166 Genetic analysis in the first family revealed two compound heterozygous variants 167 in PLEKHG5, NM_198681.3:

i) the c.1835_1860delGGCAGCGGCTGGCGGCCGTGGTGAGC (exon 16), p.Arg612Profs*111, and affecting the Rho Guanine Exchange Factor (RhoGEF) 170 domain, and ii) the c.2308delG (exon 20), p.Glu770Serfs ${ }^{\star} 72$, and affecting the 171 Pleckstrin Homology (PH) domain (Figure 1B).

172 In family 2, the two siblings were homozygous for the c.104delG (exon2), 173 p.Cys35Phefs*100, while their mother was heterozygous.

174 These variants were absent in GnomAD (Table3).

\section{DISCUSSION}

177 Rapid progress in molecular genetics, including the increasing availability of next178 generation sequencing has allowed to identify over 100 genes associated to CMT 
$179[1,12]$ and has unveiled a striking genetic and clinical overlap between CMT, distal hereditary motor neuropathies (HMN) and lower motor neuron syndromes

$181[13,14]$. Such is the case for PLEKHG5, associated with both proximal SMA, 182 intermediate CMT and HMN [3-6].

PLEKHG5 is predominantly expressed in the central and peripheral nervous system [15]. It has been shown to regulate autophagy of synaptic vesicles in axon terminals of motoneurons, its depletion leading to impaired autophagy and defective axon growth in cultured motoneurons [16]. Its RhoGEF domain involved in the activation of NK-kB signaling pathway contributes to the activation of GTPases which are in turn implicated in signaling mechanisms that regulate neuronal plasticity, axonal growth, synapse formation, actin cytoskeleton dynamics and neuronal survival[3,16-18]. The $\mathrm{PH}$ domain has been shown to regulate the RhoGEF domain. Plekhg5 inactivation in a murine model results in a late-onset motoneuron disease with degeneration of axon terminals[16] but also defective axon/Schwann cell units characterized by myelin infoldings in peripheral nerves[19]. Moreover, aggregate formation have been observed in mutant Plekhg5 murine motoneurons[3]. Finally, sural nerve biopsies in intermediate forms of PLEKHG5-related CMT have revealed severe loss of myelinated fibres [4] while they showed no abnormalities in patients presenting with the SMA form [3], suggesting different pathomechanisms might be involved.

So far, fifteen families carrying biallelic PLEKHG5 pathogenic variants have been reported [3-8]. Patients presented with either LMND (or DSMA4), in the first

201 family reported [3] and in more recently reported patients[6,7], or with a proximal and distal motor neuropathy with initial prominent proximal weakness and mild sensory involvement in 3 families [6], or with an intermediate CMT phenotype 
exhibiting significant clinical and neurophysiological sensory involvement in six 205 families [4-6].

206 We report here four additional patients from two unrelated families presenting 207 with predominantly distal weakness and variable mild sensory involvement. Age at onset was variable, ranging from early childhood in one patient to 15-20 years in the three others. Neither spinal deformities were noted, nor cranial nerve, 210 respiratory nor cardiac involvement were detected. Neurophysiological studies were compatible with an intermediate CMT but revealed in all four patients multiple conduction blocks out of typical nerve compression sites. The latter finding can sometimes hinder the diagnosis of a hereditary neuropathy, especially

214 in the absence of a known family history or when atypical clinical features are

215 present. Indeed, conduction blocks, raised CSF protein levels and the occurrence 216 of a proximal weakness may lead to discuss the diagnosis of chronic 217 inflammatory polyradiculoneuropathy leading to the prescription of intravenous 218 immunoglobulins, steroids or other immunosuppressive therapies. As such, this have been described in different forms of CMT, either dominant, X-kinked or

220 recessive: i.e. CMT4J, caused by recessive mutations in the phosphoinositide 221 phosphatase FIG4 gene CMT1B with MPZ mutations, CMT1C due to LITAF mutations, CMTX due to GJB1 mutations or CMT4C caused by recessive SH3TC mutations [20-25].

224 CK levels in two of our patients were strikingly elevated as seen in two previously reported patients[6], which may be part of the spectrum of PLEKHG5-related neuropathies. Raised CK levels have also been reported in other CMT forms such as those associated with $M P Z$ or $P M P 22$ pathogenic variants, amongst others [26-28]. Nonetheless, this may lead to the suspicion of a muscle disorder and 
229 hence to a muscle biopsy. Interestingly, brain MRI revealed diffuse white matter 230 abnormalities in patient III.5, as reported in one patient by Chen and 231 colleagues[6]. A history of muscle weakness along with raised CK levels and 232 leukoencephalopathy should raise suspicion of a muscle dystrophy due to 233 LAMA2 mutations [29,30] which have been excluded in our patient. Moreover, 234 white matter abnormalities have been classically described associated with 235 metabolic disorders such as lysosomal leukodystrophies (i.e. Krabbe or metachromatic leukodystrophy) which may present with peripheral neuropathy $237[31,32]$.

238 Central nervous system involvement and particularly diffuse white matter lesions 239 have also been reported in CMT patients with mutations in different genes, such 240 as PMP22 [33], GJB1 [34], MFN2 [35] or NEFL [36]. Interestingly, a recent study using brain diffusion tensor imaging (DTI) in a subset of 45 CMT patients has

242 shown abnormalities in 35 (including CMT1E/2A/2E/X1 forms) including 243 decreased fractional anisotropy and axial diffusivity with increased radial 244 diffusivity, suggesting white matter involvement albeit normal brain MRI imaging 245 in all patients [37].

246 PLEKHG5 is also expressed in the central nervous system [15]. Interestingly, it 247 has been found to be involved in glioma tumor cell migration and invasion [38]. 248 So far, diffuse brain white matter changes have been reported in one patient with 249 PLEKHG5 mutations [6]. We report the second case here, suggesting that 250 leukoencephalopathy may indeed be part of the clinical spectrum, but further 251 reports and experimental data are needed to confirm this observation. 
252 Genetic analysis revealed three previously unreported mutations in our patients.

253 All of them are of small frameshift deletions leading to premature termination

254

255 codons (PTC). The transcribed mRNAs from the two first fragment variants (family 1: c.1835_1860del and c.2308delG) are predicted to be degraded by the non-sense mRNA mediated decay (NMD) pathway since they lead to PTC located more than 50-55 nucleotides upstream of exon-exon junction triggering efficient NMD [39,40]. The c.104delG (p.Cys35Phefs*100) variant (exon2) is located 39 nucleotides upstream of the 3' exon 5 junction complex and thus predicted to escape the NMD machinery, and being either repressed by the nonsense-mediated translational repression system (NMTR), as reported in the literature [41], or leading to a truncated protein which is supposed to be potentially eliminated by the Ubiquitin Proteasome Pathway. Experimental studies would be needed in order to clarify this point.

Despite the different clinical phenotypes reported so far, no genotype-phenotype correlations have been identified to date. Truncating as well as missense variants affecting both main functional domains, inter-domains, $\mathrm{N}$-terminal or C-terminal parts of the protein, were associated with either CMT[4,5] or LMND (HMN, DSMA4 or SMA)[3,7,8,38]. The site of the mutation and the potential involvement of the functional protein domains or the predicted impact of the mutation have not been associated with the phenotype variability among reported families. Intrafamilial variability in terms of severity, age at onset and course of the disease, as seen in the two families reported here, has also been reported [4].

In conclusion, PLEKHG5-associated disease encompasses a continuum phenotypical spectrum from LMND (DSMA, HMN) to intermediate CMT, including 
277 also a range of motor-predominant hereditary neuropathies with proximal onset.

278 Our results expand the clinical and electrophysiological spectrum of the disease, 279 confirming diffuse white matter abnormalities and highly increased CK levels but 280 also revealing the presence of previously unreported conduction blocks on NCS. 281 The awareness and recognition of this features can contribute to a 282 better interpretation of novel PLEKHG5 genetic variants. The appropriate recognition of symptoms associated to an inherited neuropathy (motor signs during childhood, foot deformities, scoliosis, predominant distal involvement) are

clue features to distinguish acquired neuropathies such as CIDP from hereditary sensorimotor neuropathy with conduction block and thus to avoid inappropriate therapeutic strategies, specially immunosuppressive treatments.

\section{REFERENCES}

[1] Laurá M, Pipis M, Rossor AM, Reilly MM. Charcot-marie-Tooth disease and related disorders: An evolving landscape. Curr Opin Neurol

[2] Murphy SM, Laura M, Fawcett K, Pandraud A, Liu Y-T, Davidson GL, et al. Charcot-Marie-Tooth disease: frequency of genetic subtypes and guidelines for genetic testing. J Neurol Neurosurg Psychiatry 2012;83:706-10. doi:10.1136/jnnp-2012-302451.

[3] Maystadt I, Rezsöhazy R, Barkats M, Duque S, Vannuffel P, Remacle S, et al. The nuclear factor KB-activator gene PLEKHG5 is mutated in a form of autosomal recessive lower motor neuron disease with childhood onset. Am J Hum Genet 2007;81:67-76. doi:10.1086/518900.

[4] Azzedine H, Zavadakova P, Planté-Bordeneuve V, Pato MV, Pinto N, Bartesaghi L, et al. pLEKHG5 deficiency leads to an intermediate form of 
autosomal-recessive charcot-marie-tooth disease. Hum Mol Genet 2013;22:4224-32. doi:10.1093/hmg/ddt274.

305

306

[5] Kim HJ, Hong Y Bin, Park JM, Choi YR, Kim YJ, Yoon BR, et al. Mutations in the PLEKHG5 gene is relevant with autosomal recessive intermediate Charcot-Marie-Tooth disease. Orphanet J Rare Dis 2013;8. doi:10.1186/1750-1172-8-104.

[6] Chen Z, Maroofian R, Başak AN, Shingavi L, Karakaya M, Efthymiou S, et al. Novel variants broaden the phenotypic spectrum of PLEKHG5associated neuropathies. Eur J Neurol 2020:ene.14649. doi:10.1111/ene.14649.

[7] Gonzalez-Quereda L, Pagola I, Fuentes Prior P, Bernal S, Rodriguez MJ, Torné L, et al. Novel PLEKHG5 mutations in a patient with childhoodonset lower motor neuron disease. Ann Clin TransI Neurol 2020:acn3.51265. doi:10.1002/acn3.51265.

[8] Özoğuz A, Uyan Ö, Birdal G, Iskender C, Kartal E, Lahut S, et al. The distinct genetic pattern of ALS in Turkey and novel mutations. Neurobiol Aging 2015;36:1764.e9-1764.e18.

doi:10.1016/j.neurobiolaging.2014.12.032.

[9] Tankisi H, Pugdahl K, Beniczky S, Andersen H, Fuglsang-Frederiksen A. Evidence-based recommendations for examination and diagnostic strategies of polyneuropathy electrodiagnosis. Clin Neurophysiol Pract 2019;4:214-22. doi:10.1016/j.cnp.2019.10.005.

[10] Van Den Bergh PYK, Hadden RDM, Bouche P, Cornblath DR, Hahn A, Illa I, et al. European federation of neurological societies/peripheral nerve society guideline on management of chronic inflammatory demyelinating 
polyradiculoneuropathy: Report of a joint task force of the European Federation of Neurological Societies and the Peripheral Nerve Society First Revision. Eur J Neurol 2010;17:356-63. doi:10.1111/j.14681331.2009.02930.x.

[11] Dubowitz V, Sewry CA. Muscle Biopsy: a Practical Approach. Philadelphia: Saunders Elsevier; 2007.

[12] Pipis M, Rossor AM, Laura M, Reilly MM. Next-generation sequencing in Charcot-Marie-Tooth disease: opportunities and challenges. Nat Rev

[16] Lüningschrör P, Binotti B, Dombert B, Heimann P, Perez-Lara A, Slotta C, et al. Plekhg5-regulated autophagy of synaptic vesicles reveals a pathogenic mechanism in motoneuron disease. Nat Commun 2017;8. doi:10.1038/s41467-017-00689-z.

[17] Estrach S, Schmidt S, Diriong S, Penna A, Blangy A, Fort P, et al. The human Rho-GEF Trio and its target GTPase RhoG are involved in the NGF pathway, leading to neurite outgrowth. Curr Biol 2002;12:307-12. 
doi:10.1016/S0960-9822(02)00658-9.

[18] May V, Schiller MR, Eipper BA, Mains RE. Kalirin Dbl-homology guanine nucleotide exchange factor 1 domain initiates new axon outgrowths via RhoG-mediated mechanisms. J Neurosci 2002;22:6980-90. doi:10.1523/jneurosci.22-16-06980.2002.

[19] Lüningschrör P, Slotta C, Heimann P, Briese M, Weikert UM, Massih B, et al. Absence of Plekhg5 Results in Myelin Infoldings Corresponding to an Impaired Schwann Cell Autophagy, and a Reduced T-Cell Infiltration Into Peripheral Nerves. Front Cell Neurosci 2020;14. doi:10.3389/fncel.2020.00185.

[20] Yger M, Stojkovic T, Tardieu S, Maisonobe T, Brice A, Echaniz-Laguna A, et al. Characteristics of clinical and electrophysiological pattern of Charcot-Marie-Tooth 4C. J Peripher Nerv Syst 2012;17:112-22. doi:10.1111/j.1529-8027.2012.00382.x.

[21] Cottenie E, Menezes MP, Rossor AM, Morrow JM, Yousry TA, Dick DJ, et al. Rapidly progressive asymmetrical weakness in Charcot-Marie-Tooth disease type $4 \mathrm{~J}$ resembles chronic inflammatory demyelinating polyneuropathy. Neuromuscul Disord 2013;23:399-403. doi:10.1016/j.nmd.2013.01.010.

[22] Li J, Stefanelli M. Conduction Block in Charcot-Marie Tooth Neuropathy Type 4J (230). Neurology 2020;94:230.

[23] Murphy SM, Laurá M, Blake J, Polke J, Bremner F, Reilly MM. Conduction block and tonic pupils in Charcot-Marie-Tooth disease caused by a myelin protein zero p.lle112Thr mutation. Neuromuscul Disord 2011;21:223-6. doi:10.1016/j.nmd.2010.12.010. 
378 [24] Ciotti P, Luigetti M, Geroldi A, Capponi S, Pezzini I, Gulli R, et al. A novel

379

380

381

382

383

384

385

386

387

388

389

390

391

392

393

394

395

396

397

398

399

400

401

402
LITAF/SIMPLE mutation within a family with a demyelinating form of Charcot-Marie-Tooth disease. J Neurol Sci 2014;343:183-6.

doi:10.1016/j.jns.2014.05.029.

[25] Pareyson D, Scaioli V, Laurà M. Clinical and electrophysiological aspects of charcot-marie-tooth disease. NeuroMolecular Med 2006;8:3-22. doi:10.1385/NMM:8:1-2:3.

[26] Taniguchi T, Ando M, Okamoto Y, Yoshimura A, Higuchi Y, Hashiguchi A, et al. Genetic spectrum of Charcot-Marie-Tooth disease associated with myelin protein zero gene variants in Japan. Clin Genet 2021;99:359-75. doi:10.1111/cge.13881.

[27] Jordan A, Nagaraj A, Hoyle JC, Stino AM, Arnold WD, Elsheikh B. Elevated Creatinine Kinase in Peripheral Neuropathy Is Associated With Muscle Cramping. Front Neurol 2021;12. doi:10.3389/fneur.2021.613599.

[28] Hattori N, Yamamoto M, Yoshihara T, Koike H, Nakagawa M, Yoshikawa $\mathrm{H}$, et al. Demyelinating and axonal features of Charcot-Marie-Tooth disease with mutations of myelin-related proteins (PMP22, MPZ and Cx32): A clinicopathological study of 205 Japanese patients. Brain 2003;126:134-51. doi:10.1093/brain/awg012.

[29] Sarkozy A, Foley AR, Zambon AA, Bönnemann CG, Muntoni F. LAMA2Related Dystrophies: Clinical Phenotypes, Disease Biomarkers, and Clinical Trial Readiness. Front Mol Neurosci 2020;13. doi:10.3389/fnmol.2020.00123.

[30] Vainzof M, Marie SKN, Reed UC, Schwartzmann JS, Pavanello RCM, Passos-Bueno MR, et al. Deficiency of merosin (laminin M or a2) in 
congenital muscular dystrophy associated with cerebral white matter alterations. Neuropediatrics 1995;26:293-7. doi:10.1055/s-2007-979777.

[31] Kohlschütter A. Lysosomal leukodystrophies. Krabbe disease and metachromatic leukodystrophy. Handb. Clin. Neurol., vol. 113, Elsevier B.V.; 2013, p. 1611-8. doi:10.1016/B978-0-444-59565-2.00029-0.

[32] Raina A, Nair S, Nagesh C, Thomas B, Nair M, Sundaram S. Electroneurography and advanced neuroimaging profile in pediatric-onset metachromatic leukodystrophy. J Pediatr Neurosci 2019;14:70-5. doi:10.4103/jpn.JPN_155_18.

[33] Koros C, Evangelopoulos M-E, Kilidireas C, Andreadou E. Central Nervous System Demyelination in a Charcot-Marie-Tooth Type 1A Patient. Case Rep Neurol Med 2013;2013:1-4. doi:10.1155/2013/243652.

[34] Paulson HL, Garbern JY, Hoban TF, Krajewski KM, Lewis RA, Fischbeck $\mathrm{KH}$, et al. Transient central nervous system white matter abnormality in Xlinked Charcot-Marie-Tooth disease. Ann Neurol 2002;52:429-34. doi:10.1002/ana.10305.

[35] Brockmann K, Dreha-Kulaczewski S, Dechent P, Bönnemann C, Helms G, Kyllerman M, et al. Cerebral involvement in axonal Charcot-MarieTooth neuropathy caused by mitofusin 2 mutations. J Neurol 2008;255:1049-58. doi:10.1007/s00415-008-0847-1.

[36] Berciano J, Peeters K, García A, López-Alburquerque T, Gallardo E, Hernández-Fabián A, et al. NEFL N98S mutation: another cause of dominant intermediate Charcot-Marie-Tooth disease with heterogeneous early-onset phenotype. J Neurol 2016;263:361-9. doi:10.1007/s00415015-7985-z. 
[37] Lee M, Park CH, Chung HK, Kim HJ, Choi Y, Yoo JH, et al. Cerebral white matter abnormalities in patients with charcot-marie-tooth disease. Ann Neurol 2017;81:147-51. doi:10.1002/ana.24824.

[38] Qian M, Chen Z, Wang S, Guo X, Zhang Z, Qiu W, et al. PLEKHG5 is a novel prognostic biomarker in glioma patients. Int J Clin Oncol 2019;24:1350-8. doi:10.1007/s10147-019-01503-0.

[39] Dyle MC, Kolakada D, Cortazar MA, Jagannathan S. How to get away with nonsense: Mechanisms and consequences of escape from nonsense-mediated RNA decay. Wiley Interdiscip Rev RNA 2020;11. doi:10.1002/wrna.1560.

[40] Nagy E, Maquat LE. A rule for termination-codon position within introncontaining genes: When nonsense affects RNA abundance. Trends Biochem Sci 1998;23:198-9. doi:10.1016/S0968-0004(98)01208-0.

[41] Kwon TY, Long SL, Kim NG, Hyun JK, Kwi HK, Chwae YJ, et al. Selective translational repression of truncated proteins from frameshift mutationderived mRNAs in tumors. PLoS Biol 2007;5:1098-109. doi:10.1371/journal.pbio.0050109. 
447 FIGURE LEGENDS

448 Table 1. Summarized clinical and ancillary tests findings.

449 a (-) no weakness, (+) 4/5 on Medical Research Council (MRC) scale, $(++)<4 / 5$

450 on MRC scale, $(+++)$ complete paralysis; ${ }^{b}(-)$ no atrophy, (+) mild atrophy, $(++)$

451 moderate atrophy, $(+++)$ severe atrophy; ${ }^{c}(-)$ normal, $(+)$ mild hypoesthesia, $(++)$

452 profound hypoesthesia; ${ }^{\mathrm{d}}(+)$ normal, $(+/-)$ decreased, (-) areflexia; CK= creatin

453 kinase; $C S F=C e r e b r o s p i n a l$ fluid; $L L=$ Lower limbs; $N=$ normal values; $N A=$ no 454 data available; NCS= nerve conduction studies; UL= upper limbs; $\mathrm{U} / \mathrm{L}=$ units/liter 455

Table 2. Summarized EMG findings. ${ }^{a}$ Motor nerve conduction studies (MNCS).

457 CMAP: compound motor action potentials $(\mathrm{mV})$. Muscles recorded: median: 458 abductor pollicis brevis (APB) / ulnar: adductor digiti minimi / superficial peroneal: 459 extensor digitorum brevis / tibial: tibialis posterior. AE: above-elbow. BE: below 460 elbow. BFH: below fibular head. PF: popliteal fossa. Normal CMAP values: Median-Ulnar > 6; Peroneal $>3$; Tibial $>6$. Conduction blocks are indicated in bold type. MNCV: motor nerve conduction velocity $(\mathrm{m} / \mathrm{s})$. Normal MNCV values: Median-Ulnar > 42; Peroneal-Tibial 42. Distal latencies and $\mathrm{F}$ latencies are indicated in ms. b Sensory nerve conduction studies (SNCS). SNAP: sensory 465 nerve action potential $(\mu \mathrm{V})$. Normal SNAP values: Median $>15$; Ulnar $>8$; Superficial peroneal-Sural > 10. SNCV: Sensory conduction velocities $(\mathrm{m} / \mathrm{s}$; 467 orthodromic). SNCV normal values= Median-Ulnar $>45$; Superficial peroneal468 Sural 40.

$469 \quad \mathrm{NA}=$ not ascertained. $\mathrm{NR}=$ not recordable

470

471 
472 Table 3. Summarized information of the reported novel variants. Het:

473 heterozygous, Hom: homozygous; NGS: next generation sequencing; WES:

474 whole exome sequencing

475

476 Figure 1. (A) Family pedigrees. Black-filled symbols: affected individuals. Black-

477 arrows: index cases. Electropherograms indicating the corresponding genetic

478 variants. (B) Schematic representation of the PLEKHG5 genomic sequence

479 (upper panel) and protein (lower panel), including the main functional

domains, based on NCBI reference sequence NM_198681.3. Localization of

the novel mutations reported here (red) and the previously reported mutations

482 (black). PH: Pleckstrin Homology domain. RBD: Ras Binding Domain.

483 RhoGEF : Rho Guanine Exchange Factor domain.

Figure 2. Neurophysiological and imaging findings. (A) Conduction block on ulnar motor NCS recorded on abductor digiti minimi in patient III.5 from family 1.

(B) Multiple conduction blocs on UL in patient II.4 from family 2. Conduction block on median nerve (recorded on abductor pollicis brevis) (A) and ulnar nerves (recorded on abductor digiti minimi) (B). Note reduced CMAP amplitude on proximal (elbow) stimulation compared with distal (wrist) stimulation. (C-D)

Needle EMG of gastrocnemius muscle from patient III.4 from family 1 showing complex repetitive discharges $(C)$ along with giant MUAPs and poor recruitment 493 indicating a chronic neurogenic pattern. (E): Brain MRI from patient 2-II.4. T2 FLAIR-weighted axial images reveal diffuse and bilateral periventricular white matter lesions (white arrows). (F-G): Note distal wasting, cavus feet and hammer 
497 weighted) reveals mild fatty degeneration affecting quadriceps and sartorius in 498 thighs. Note prominent atrophy and fatty degeneration of gastrocnemius and 499 soleus muscles.

500 